\section{Non-Fragile Exponential Stability Assignment of Discrete-Time Linear Systems With Missing Data in Actuators}

\author{
Zhan Shu, James Lam, and Junlin Xiong
}

\begin{abstract}
This technical note is concerned with the non-fragile exponential stabilization for a class of discrete-time linear systems with missing data in actuators. The process of missing data is modeled by a discrete-time Markov chain with two state components. When no uncertainty exists in the controllers, a necessary and sufficient condition, which not only guarantees the exponential stability but also gives a lower bound on the decay rate, is established in terms of linear matrix inequalities (LMIs). Based on this condition, an LMI-based approach is provided to design a non-fragile state-feedback controller such that the closed-loop system is exponentially stable with a prescribed lower bound on the decay rate for the known missing data process and all admissible uncertainties in controllers. A numerical example is provided to show the effectiveness of the theoretical results.
\end{abstract}

Index Terms-Exponential stability, linear matrix inequality (LMI), Markov chain, missing data, non-fragile control.

\section{INTRODUCTION}

Stability and stabilization of dynamic systems have always been the essential issues in control theory and engineering. Over decades, a lot of effort has been devoted to this area, and a large number of synthesis approaches have been provided to design stabilizing controllers [1]-[4]. An implicit assumption inherent in these approaches is that the controllers will be implemented exactly. However, in practice, controllers may have a certain degree of errors owing to finite word length in digital systems, the imprecision inherent in analog systems and the need for additional tuning of parameters in the final controller implementation. Hence, how to design a controller insensitive to the variations in its gain, i.e., the controller is non-fragile, has received much attention. The stabilization problem of discrete-time linear systems with guaranteed cost has been studied in [5], [6]. In [7], the design problem of non-fragile guaranteed cost controllers for uncertain descriptor systems with delays has been investigated thoroughly. Recently, some results on the stabilization and $\mathcal{H}_{\infty}$ control for uncertain stochastic time-delay systems have been provided in [8].

In the literature mentioned above, it is assumed that the outputs of actuators always contain signals. However, in practical applications, there may be a nonzero probability that the outputs of actuators do not consist of any signal, i.e., the signals contain missing data. The missing data may be caused by a variety of reasons, e.g., the uncertain deadzone nonlinearity in actuators, intermittent actuator failures, a certain failure in the data transmission, or some of the data may be jammed or coming from a high noisy environment. For example, if the controller signals

Manuscript received December 22, 2006; revised September 21, 2007. Current version published March 11, 2009. This work was supported in part by RGC 7031/07P. Recommended by Associate Editor M. Kothare.

Z. Shu and J. Lam are with the Department of Mechanical Engineering, The University of Hong Kong, Hong Kong, China (e-mail: shuzhan @hku.hk; james. lam@hku.hk).

J. Xiong is with School of Information Technology and Electrical Engineering, University of New South Wales at the Australian Defence Force Academy, Canberra ACT 2600, Australia (e-mail: junlin.xiong@gmail.com).

Color versions of one or more of the figures in this technical note are available online at http://ieeexplore.ieee.org.

Digital Object Identifier 10.1109/TAC.2008.2009598 are transmitted through the so-called Gilbert-Elliott channel [9], [10], then the data may be lost in a process governed by a two-state Markov chain. Some results on the estimation and filtering of linear systems with missing data can be found in [11]-[14]. However, to the authors' knowledge, little results are available for the exponential stability assignment problem of linear systems with missing data, especially for the case with uncertain controllers.

In this technical note, we study the stabilization problem of discrete-time linear systems with uncertainties in controllers and missing data in actuators. A necessary and sufficient condition with the decay rate constraint is established for the case without controller uncertainties in terms of LMIs. Based on this condition, a state-feedback controller is designed such that the closed-loop system is exponentially stable with a prescribed lower bound on the decay rate for all admissible controller uncertainties and the known missing data process. A numerical example is provided to show the effectiveness of the proposed approach.

Notation: Throughout this technical note, $\mathbb{R}^{n}, \mathbb{C}^{n}, \mathbb{R}^{m \times n}, \mathbb{C}^{m \times n}$ represent the $n$-dimensional Euclidean space, the $n$-dimensional complex vector space, the set of all $m \times n$ real matrices, and the set of all $m \times n$ complex matrices, respectively; the superscript " $T$ " and "*" represent the transpose and the conjugate transpose, respectively; for Hermitian matrices $X=X^{*} \in \mathbb{C}^{n \times n}$ and $Y=Y^{*} \in \mathbb{C}^{n \times n}$, the notation $X \geq Y$ (respectively, $X>Y$ ) means that the matrix $X-Y$ is positive semi-definite (respectively, positive definite); we denote $\left(\mathbb{C}^{n}\right)^{+}=\left\{L \in \mathbb{C}^{n \times n} ; L=L^{*} \geq 0\right\} ; I$ is the identity matrix with appropriate dimension; $\mathbf{E}\{\cdot\}$ denotes the expectation operator with respect to some probability measure; $\|\cdot\|$ represents the Euclidean norm for a vector, and the spectral norm for a matrix; $|\cdot|$ denotes the module of a complex number $x$, i.e., $|x|=\sqrt{x^{*} x} ; \rho(\cdot)$ stands for the spectral radius of a matrix; the symbol $\otimes$ denotes the Kronecker Product; associated with a matrix $H \in \mathbb{C}^{m \times n}$, the column operator $\varphi(\cdot)$ is defined as

$$
\varphi(H)=\left[h_{11}, \ldots, h_{m 1}, h_{12}, \ldots, h_{m 2}, \ldots, h_{1 n}, \ldots, h_{m n}\right]^{T} ;
$$

associated with a set of matrices $H_{i} \in \mathbb{C}^{m \times n}, i=1,2, \ldots, N$, the column operator $\hat{\varphi}(\cdot)$ is defined as

$$
\hat{\varphi}\left(H_{1}, \ldots, H_{N}\right)=\left[\begin{array}{llll}
\varphi^{T}\left(H_{1}\right) & \varphi^{T}\left(H_{2}\right) & \cdots & \varphi^{T}\left(H_{N}\right)
\end{array}\right]^{T} ;
$$

associated with a set of matrices $A_{i} \in \mathbb{C}^{n \times n}, i=1,2, \ldots, N$, $\operatorname{diag}\left(A_{1}, A_{2}, \ldots, A_{N}\right)$ is defined as

$$
\operatorname{diag}\left(A_{1}, A_{2}, \ldots, A_{N}\right)=\left[\begin{array}{ccc}
A_{1} & \cdots & 0 \\
\vdots & \ddots & \vdots \\
0 & \cdots & A_{N}
\end{array}\right] ;
$$

the symbol \# is used to denote a matrix which can be inferred by symmetry. Matrices, if their dimensions are not explicitly stated, are assumed to have compatible dimensions for algebraic operations.

\section{PRELIMINARIES AND PROBLEM Formulation}

Consider the following class of discrete-time linear systems with missing data in actuators:

$$
x(k+1)=A x(k)+\gamma(k) B u(k)
$$

where $x(k) \in \mathbb{R}^{n}$ and $u(k) \in \mathbb{R}^{m}$ are the system state and the control input, respectively, and $A$ and $B$ are known constant matrices; the parameter $\gamma(k)$ represents the possible missing data process in actuators, and it is assumed to be a discrete-time homogeneous Markov chain 
taking values in a finite set $\mathcal{S}=\{0,1\}$ with transition probability matrix

$$
\Pi=\left[\begin{array}{cc}
1-\alpha & \alpha \\
\beta & 1-\beta
\end{array}\right]
$$

where $0 \leq \alpha=\operatorname{Pr}(\gamma(k+1)=1 \mid \gamma(k)=0) \leq 1$ and $0 \leq \beta=$ $\operatorname{Pr}(\gamma(k+1)=0 \mid \gamma(k)=1) \leq 1$ are called the recovery rate and the failure rate. When $\alpha+\beta=1, \gamma(k)$ reduces to the Bernoullitype missing data process, which is considered in [11], [15], with the probability distribution

$$
\operatorname{Pr}(\gamma(k)=1)=\alpha, \quad \operatorname{Pr}(\gamma(k)=0)=1-\alpha .
$$

In this technical note, we consider the following controller form:

$$
u(k)=(K+\Delta K) x(k)
$$

where $\Delta K$ represents possible controller gain fluctuation. It is assumed that $\Delta K$ has the following structure:

$$
\Delta K=\left[\begin{array}{ccc}
k_{11}\left(\delta_{1}+\delta_{11}\right) & \cdots & k_{1 n}\left(\delta_{1}+\delta_{1 n}\right) \\
k_{21}\left(\delta_{2}+\delta_{21}\right) & \cdots & k_{2 n}\left(\delta_{2}+\delta_{2 n}\right) \\
\vdots & \ddots & \vdots \\
k_{m 1}\left(\delta_{m}+\delta_{m 1}\right) & \cdots & k_{m n}\left(\delta_{m}+\delta_{m n}\right)
\end{array}\right]
$$

where $\delta_{i}$ and $\delta_{i j}$ are real uncertain parameters satisfying

$$
\left|\delta_{i}\right| \leq \bar{\delta}_{1 i} \leq 1, \quad\left|\delta_{i j}\right| \leq \bar{\delta}_{i j} \leq \bar{\delta}_{2 j} \leq 1
$$

Then, $\Delta K$ can be re-written in a compact form

$$
\Delta K=\Phi K+\sum_{i=1}^{m} \sum_{j=1}^{n} \delta_{i j} E_{i} K H_{j}
$$

where

$$
\Phi=\operatorname{diag}\left(\delta_{1}, \delta_{2}, \ldots \delta_{m}\right)
$$

and $E_{i} \in \mathbb{R}^{m \times m}, H_{j} \in \mathbb{R}^{n \times n}$ are rank-one matrices with entry ' 1 ' located at the $i$ th and the $j$ th position of the main diagonal, respectively.

Remark 1: The multiplicative gain variation model $\Delta K$ of the form in (6) was first introduced in [16]. When $\delta_{i j} \equiv 0, \Delta K$ reduces to the degradation model of actuators [17]. If $\delta_{i} \equiv 0$, the model $\Delta K$ of (6) represents more general multiplicative gain variations proposed in [18]. In practice, this model can be used to represent actuator degradation, controller implementation errors, such as round-off error, quantization errors, controller realization errors, and can also deal with the practical issue of controller tuning, such as improper initialization of the controller [16], [19].

When a controller in (3) is applied to (1), the resulting closed-loop system becomes

$$
x(k+1)=[A+\gamma(k) B(K+\Delta K)] x(k) .
$$

Throughout the technical note, we use the following definitions for the closed-loop system in (8).

Definition 1:

1) For a scalar $\lambda \geq 1$, the closed-loop system in (8) with $\Delta K \equiv 0$ is said to be $\lambda$-exponentially stable if there exist scalars $\varepsilon>0$ and $\sigma>0$ such that

$$
\mathbf{E}\left\{\|x(k)\|^{2}\right\} \leq \sigma(\lambda+\varepsilon)^{-k}\left\|x_{0}\right\|^{2}
$$

where $\lambda+\varepsilon$ is called the decay rate, namely, the system possessing a decay rate larger than $\lambda$.
2) The closed-loop system in (8) is said to be reliably $\lambda$-exponentially stable if it is $\lambda$-exponentially for all possible uncertainties in (5).

Our goal is to design a controller matrix $K$ such that the closed-loop system is reliably $\lambda$-exponentially stable for a prescribed constant $\lambda \geq$ 1 . We end this section by giving several lemmas which will be useful in the sequel.

Lemma 1 ([20], [21]): For any $L \in \mathbb{C}^{n \times n}$, there exist matrices $L_{1}, L_{2}, L_{3}, L_{4} \in\left(\mathbb{C}^{n}\right)^{+}$such that $L=\left(L_{1}-L_{2}\right)+\sqrt{-1}\left(L_{3}-L_{4}\right)$.

Lemma 2: For any $P \in\left(\mathbb{C}^{n}\right)^{+}$, there exist matrices $P_{i}=x_{i} x_{i}^{*}$, $i=1,2, \ldots, n$, with $x_{i} \in \mathbb{C}^{n}$ such that $P=\sum_{i=1}^{n} P_{i}$.

Lemma 3: For any $X, Y \in\left(\mathbb{C}^{n}\right)^{+}$, if $X \geq Y$, then $\|X\| \geq\|Y\|$.

Lemma 4 ([22], [23]): Let $G$ and $Q$ be real symmetric matrices and $M, N$ be real matrices with appropriate dimensions. Then, For $Q>0$, we have $M N+(M N)^{T} \leq M Q M^{T}+N^{T} Q^{-1} N$.

Lemma 5 ([24]): For the column operator $\varphi(\cdot)$, we have the following properties:

1) $\varphi(a G+b H)=a \varphi(G)+b \varphi(H)$, for $G, H \in \mathbb{C}^{m \times n}, a, b \in \mathbb{C}$.

2) $\varphi(G X H)=\left(H^{T} \otimes G\right) \varphi(X)$, for $G, X, H \in \mathbb{C}^{n \times n}$.

Lemmas 2 and 3 can be proved readily by employing singular value decomposition.

\section{III. $\lambda$-EXPONENTIAL STABILITY ANALYSIS}

We provide a necessary and sufficient condition on $\lambda$-exponential stability of the closed-loop system without controller uncertainties in the following theorem.

Theorem 1: For a prescribed scalar $\lambda \geq 1$, the closed-loop system in (8) with $\Delta K \equiv 0$ is $\lambda$-exponentially stable if and only if there exist real matrices $P_{0}>0$ and $P_{1}>0$ such that

$$
\begin{aligned}
\lambda A^{T} \hat{P}_{0} A-P_{0} & <0, \\
\lambda(A+B K)^{T} \hat{P}_{1}(A+B K)-P_{1} & <0,
\end{aligned}
$$

where

$$
\begin{aligned}
& \hat{P}_{0}=(1-\alpha) P_{0}+\alpha P_{1} \\
& \hat{P}_{1}=\beta P_{0}+(1-\beta) P_{1} .
\end{aligned}
$$

Proof: (Sufficiency) It follows from (9) and (10) that there exists a small enough scalar $\varepsilon>0$ such that

$$
\begin{array}{r}
(\lambda+\varepsilon) A^{T} \hat{P}_{0} A-P_{0}<0, \\
(\lambda+\varepsilon)(A+B K)^{T} \hat{P}_{1}(A+B K)-P_{1}<0 .
\end{array}
$$

Choose a stochastic Lyapunov function candidate as follows:

$$
V(x(k), \gamma(k), k)=(\lambda+\varepsilon)^{k} x^{T}(k) P_{\gamma(k)} x(k) .
$$

Then the difference of the function along the solution of the system, for each $i \in \mathcal{S}$, is evaluated as

$$
\begin{aligned}
\Delta V & (x(k), i, k) \\
& =\mathbf{E}\{V(x(k+1), \gamma(k+1)), k+1\}-V(x(k), i, k) \\
& =(\lambda+\varepsilon)^{k} x^{T}(k)\left[(\lambda+\varepsilon) A_{i}^{T} \hat{P}_{i} A_{i}-P_{i}\right] x(k)
\end{aligned}
$$

where $A_{0}=A, A_{1}=A+B K, i \in \mathcal{S}$. From (11) and (12), we obtain that

$$
\Delta V(x(k), i, k)<0, \text { for each } i \in \mathcal{S} \text {. }
$$

Therefore, it is easy to show that

$$
\begin{aligned}
\mathbf{E}\{V(x(k), \gamma(k), k)\} & \leq V(x(0), \gamma(0), 0) \\
& \leq \max \left\{\left\|P_{0}\right\|,\left\|P_{1}\right\|\right\}\left\|x_{0}\right\|^{2} .
\end{aligned}
$$


On the other hand

$$
\mathbf{E}\left\{x^{T}(k) P_{\gamma(k)} x(k)\right\} \geq \frac{\mathbf{E}\left\{\|x(k)\|^{2} \mid x_{0}, \gamma_{0}\right\}}{\max \left\{\left\|P_{0}^{-1}\right\|,\left\|P_{1}^{-1}\right\|\right\}} .
$$

Combining (13) and (14) yields that

$$
\mathbf{E}\left\{\|x(k)\|^{2}\right\} \leq \sigma(\lambda+\varepsilon)^{-k}\left\|x_{0}\right\|^{2}
$$

where $\sigma=\max \left\{\left\|P_{0}^{-1}\right\|,\left\|P_{1}^{-1}\right\|\right\} \times \max \left\{\left\|P_{0}\right\|,\left\|P_{1}\right\|\right\}$. This proves the sufficiency.

(Necessity) If the closed-loop system with $\Delta K \equiv 0$ is $\lambda$-exponentially stable for all initial conditions $x(0)=x_{0} \in \mathbb{R}^{n}$ and $\gamma(0)=$ $\gamma_{0} \in \mathcal{S}$, then it is also $\lambda$-exponentially stable for all initial conditions $x(0)=x_{0} \in \mathbb{C}^{n}$ and $\gamma(0)=\gamma_{0} \in \mathcal{S}$. It can be shown easily that the second moment $X(k)=\mathbf{E}\left\{x(k) x^{*}(k)\right\} \in\left(\mathbb{C}^{n}\right)^{+}$, for all initial conditions $x(0)=x_{0} \in \mathbb{C}^{n}$ and $\gamma(0)=\gamma_{0} \in \mathcal{S}$, is also $\lambda$-exponentially stable. That is

$$
\begin{aligned}
\|X(k)\| & =\left\|\mathbf{E}\left\{x(k) x^{*}(k)\right\}\right\| \\
& \leq \mathbf{E}\left\{\left\|x(k) x^{*}(k)\right\|\right\} \\
& \leq \mathbf{E}\left\{\|x(k)\|^{2}\right\} \\
& \leq \sigma(\lambda+\varepsilon)^{-k}\left\|x_{0}\right\|^{2} \\
& =\sigma(\lambda+\varepsilon)^{-k}\|\varphi(X(0))\|
\end{aligned}
$$

where we use the following relationship, for $x(0)$ $\left[x_{1}(0), x_{2}(0), \ldots, x_{n}(0)\right]^{T}$

$$
\begin{aligned}
\varphi^{*}(X(0)) \varphi(X(0)) & =\sum_{j=1}^{n} \sum_{i=1}^{n}\left(\left|x_{i}(0)\right|\left|x_{j}(0)\right|\right)^{2} \\
& =\left(\sum_{i=1}^{n}\left|x_{i}(0)\right|^{2}\right)^{2} .
\end{aligned}
$$

Define

$$
X_{i}(k)=\mathbf{E}\left\{x(k) x^{*}(k)\right\} \times \mathbf{1}_{\{\gamma(k)=i\}} \in\left(\mathbb{C}^{n}\right)^{+}, \quad i \in \mathcal{S}
$$

where $\mathbf{1}_{\{.\}}$stands for the Dirac measure. It is obvious that

$$
X(k)=X_{0}(k)+X_{1}(k) .
$$

With this and Lemma 3, we obtain that

$$
\left\|X_{i}(k)\right\| \leq \sigma(\lambda+\varepsilon)^{-k}\|\varphi(X(0))\|, \quad i \in \mathcal{S} .
$$

Thus, we have that

$$
\begin{aligned}
\left\|\varphi\left(X_{i}(k)\right)\right\| & =\sqrt{\operatorname{trace}\left(X_{i}^{2}(k)\right)} \leq \sqrt{n}\left\|X_{i}(k)\right\| \\
& \leq \sqrt{n} \sigma(\lambda+\varepsilon)^{-k}\|\varphi(X(0))\|, \quad i \in \mathcal{S} .
\end{aligned}
$$

Hence

$$
\begin{aligned}
\left\|\hat{\varphi}\left(X_{0}(k), X_{1}(k)\right)\right\| & =\sqrt{\left\|\varphi\left(X_{0}(k)\right)\right\|^{2}+\left\|\varphi\left(X_{1}(k)\right)\right\|^{2}} \\
& \leq \sqrt{2 n} \sigma(\lambda+\varepsilon)^{-k}\|\varphi(X(0))\| .
\end{aligned}
$$

Through some algebraic manipulations, we have that

$X_{0}(k+1)=(1-\alpha) A X_{0}(k) A^{T}+\beta[A+B K] X_{1}(k)[A+B K]^{T}$.

Applying Lemma 5 to (18) yields that

$$
\begin{aligned}
\varphi\left(X_{0}(k+1)\right)= & (1-\alpha)(A \otimes A) \varphi\left(X_{0}(k)\right) \\
& +\beta((A+B K) \otimes(A+B K)) \varphi\left(X_{1}(k)\right) .
\end{aligned}
$$

Likewise, we have that

$$
\begin{aligned}
& \varphi\left(X_{1}(k+1)\right)=\alpha(A \otimes A) \varphi\left(X_{0}(k)\right) \\
& +(1-\beta)((A+B K) \otimes(A+B K)) \varphi\left(X_{1}(k)\right) .
\end{aligned}
$$

Therefore, from (19) and (20), $\hat{\varphi}\left(X_{0}(k), X_{1}(k)\right)$ satisfies the following difference equation:

$$
\hat{\varphi}\left(X_{0}(k+1), X_{1}(k+1)\right)=\mathcal{M}(\alpha, \beta) \hat{\varphi}\left(X_{0}(k), X_{1}(k)\right)
$$

where

$$
\mathcal{M}(\alpha, \beta)=\left(\Pi^{T} \otimes I_{n^{2}}\right) \operatorname{diag}(A \otimes A,(A+B K) \otimes(A+B K)) .
$$

If we set $\gamma(0)=0, x(0)=x_{0} \in \mathbb{C}^{n}$, then, for any $X_{0}(0)$ satisfying $X_{0}(0)=x_{0} x_{0}^{*}$

$$
\begin{aligned}
& \left\|\hat{\varphi}\left(X_{0}(k), X_{1}(k)\right)\right\| \\
& \quad \leq \sqrt{2 n} \sigma(\lambda+\varepsilon)^{-k}\|\varphi(X(0))\| \\
& \quad=\sqrt{2 n} \sigma(\lambda+\varepsilon)^{-k}\left\|\hat{\varphi}\left(X_{0}(0), X_{1}(0)=0\right)\right\| .
\end{aligned}
$$

Likewise, we have that, for any $X_{1}(0)$ satisfying $X_{1}(0)=x_{1} x_{1}^{*}$ with $x_{1} \in \mathbb{C}^{n}$,

$$
\begin{aligned}
\left\|\hat{\varphi}\left(X_{0}(k), X_{1}(k)\right)\right\| \leq \sqrt{2 n} \sigma(\lambda & +\varepsilon)^{-k} \\
& \times\left\|\hat{\varphi}\left(X_{0}(0)=0, X_{1}(0)\right)\right\| .
\end{aligned}
$$

It follows from (21) and (22) that for any initial condition $\hat{\varphi}\left(X_{0}(0), X_{1}(0)\right)$ satisfying $X_{0}(0)=x_{0} x_{0}^{*}$ and $X_{1}(0)=x_{1} x_{1}^{*}$ with $x_{0} \in \mathbb{C}^{n}$ and $x_{1} \in \mathbb{C}^{n}$ being arbitrary,

$$
\left\|\hat{\varphi}\left(X_{0}(k), X_{1}(k)\right)\right\| \leq 2 \sqrt{2 n} \sigma(\lambda+\varepsilon)^{-k}\left\|\hat{\varphi}\left(X_{0}(0), X_{1}(0)\right)\right\| .
$$

Since

$$
\hat{\varphi}\left(X_{0}(k), X_{1}(k)\right)=\mathcal{M}^{k}(\alpha, \beta) \hat{\varphi}\left(X_{0}(0), X_{1}(0)\right)
$$

we obtain from (23) that, for any initial condition $\hat{\varphi}\left(X_{0}(0), X_{1}(0)\right)$ satisfying $X_{0}(0)=x_{0} x_{0}^{*}$ and $X_{1}(0)=x_{1} x_{1}^{*}$ with $x_{0} \in \mathbb{C}^{n}$ and $x_{1} \in \mathbb{C}^{n}$ being arbitrary,

$$
\begin{aligned}
\left\|\mathcal{M}^{k}(\alpha, \beta) \hat{\varphi}\left(X_{0}(0), X_{1}(0)\right)\right\| & \\
\leq & 2 \sqrt{2 n} \sigma(\lambda+\varepsilon)^{-k}\left\|\hat{\varphi}\left(X_{0}(0), X_{1}(0)\right)\right\| .
\end{aligned}
$$

Next, we will show that $\rho(\mathcal{M}(\alpha, \beta))<\lambda^{-1}$. Assume that there exists an eigenvalue $\mu$ of $\mathcal{M}(\alpha, \beta)$ such that $|\mu| \geq \lambda^{-1}$, and $\xi_{0}=$ $\left[\xi_{01}^{T}, \xi_{02}^{T}\right]^{T}, \xi_{01}, \xi_{02} \in \mathbb{C}^{n^{2}}$ is a corresponding eigenvector, and consider the following difference equation:

$$
\xi(k+1)=\mathcal{M}(\alpha, \beta) \xi(k) .
$$

For the initial condition $\xi(0)=\xi_{0}$, there must exist $L^{(1)}, L^{(2)} \in \mathbb{C}^{n \times n}$ such that $\hat{\varphi}\left(L^{(1)}, L^{(2)}\right)=\xi_{0}$. For $L^{(\nu)} \in \mathbb{C}^{n \times n}, \nu=1,2$, by Lemma 1 , there exist $L_{i}^{(\nu)} \in\left(\mathbb{C}^{n}\right)^{+}, \nu=1,2, i=1,2,3,4$ such that

$$
L^{(\nu)}=\left(L_{1}^{(\nu)}-L_{2}^{(\nu)}\right)+\sqrt{-1}\left(L_{3}^{(\nu)}-L_{4}^{(\nu)}\right) .
$$

Furthermore, by Lemma 2, for any $L_{i}^{(\nu)} \in\left(\mathbb{C}^{n}\right)^{+}, \nu=1,2, i=$ $1,2,3,4$, there exist $L_{i 1}^{(\nu)}, L_{i 2}^{(\nu)}, \ldots, L_{i n}^{(\nu)}$ such that $L_{i}^{(\nu)}=\sum_{j=1}^{n} L_{i j}^{(\nu)}$, where $L_{i j}^{(\nu)}=x_{i j}^{(\nu)}\left(x_{i j}^{(\nu)}\right)^{*}$. On one hand, for the initial condition $\xi(0)=\xi_{0}$, we have

$$
\begin{aligned}
\xi(k) & =\mathcal{M}^{k}(\alpha, \beta) \hat{\varphi}\left(L^{(1)}, L^{(2)}\right) \\
& =\mathcal{M}^{k}(\alpha, \beta)
\end{aligned}
$$




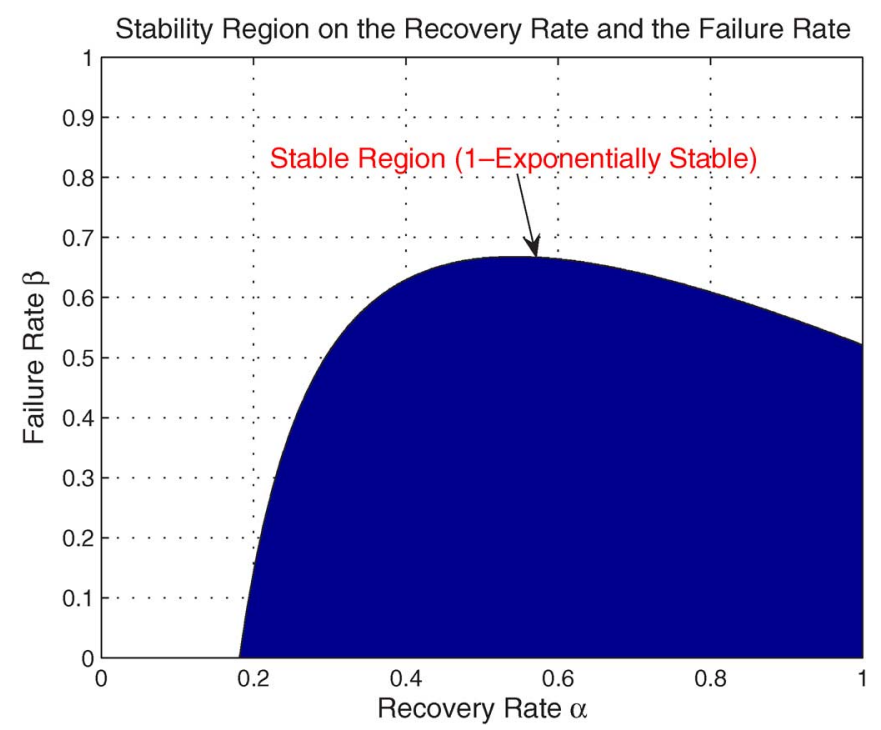

Fig. 1. Stable region on $\boldsymbol{\alpha}$ and $\boldsymbol{\beta}$.

$$
\begin{aligned}
\times \sum_{j=1}^{n}\left(\left[\begin{array}{l}
\varphi\left(L_{1 j}^{(1)}\right) \\
\varphi\left(L_{1 j}^{(2)}\right)
\end{array}\right]-\left[\begin{array}{l}
\varphi\left(L_{2 j}^{(1)}\right) \\
\varphi\left(L_{2 j}^{(2)}\right)
\end{array}\right]\right. \\
\left.+\sqrt{-1}\left(\left[\begin{array}{l}
\varphi\left(L_{3 j}^{(1)}\right) \\
\varphi\left(L_{3 j}^{(2)}\right)
\end{array}\right]-\left[\begin{array}{l}
\varphi\left(L_{4 j}^{(1)}\right) \\
\varphi\left(L_{4 j}^{(2)}\right)
\end{array}\right]\right)\right)
\end{aligned}
$$

From (24) and (25), we obtain that

$$
\|\xi(k)\| \leq c(\lambda+\varepsilon)^{-k}\left\|\xi_{0}\right\|
$$

where $c=8 \sqrt{2 n} \sigma n$. On the other hand, since $\xi_{0}$ is an eigenvector corresponding to the eigenvalue $\mu$, we have that

$$
\|\xi(k)\|=|\mu|^{k}\left\|\xi_{0}\right\|
$$

which implies that there must exist a large enough $\bar{k}>0$ such that

$$
\|\xi(\bar{k})\| \geq c(\lambda+\varepsilon)^{-\bar{k}}\left\|\xi_{0}\right\| .
$$

This contradicts with (26). Therefore, $\rho(\mathcal{M}(\alpha, \beta))<\lambda^{-1}$, which is equivalent to $\rho(\lambda \mathcal{M}(\alpha, \beta))<1$. In the following part, we show that there exist real matrices $Q_{0}>0, P_{0}>0, Q_{1}>0, P_{1}>0$ such that

$$
\begin{aligned}
\lambda A^{T} \hat{P}_{0} A-P_{0} & =-Q_{0}<0, \\
\lambda(A+B K)^{T} \hat{P}_{1}(A+B K)-P_{1} & =-Q_{1}<0 .
\end{aligned}
$$

Using the operator $\hat{\varphi}(\cdot)$ and Lemma 5, (27) and (28) can be rewritten as

$$
\left[I-\lambda \mathcal{M}^{T}(\alpha, \beta)\right] \hat{\varphi}\left(P_{0}, P_{1}\right)=\hat{\varphi}\left(Q_{0}, Q_{1}\right)
$$

It follows from $\rho(\lambda \mathcal{M}(\alpha, \beta))<1$ that, for any real matrices $Q_{0}>0$, $Q_{1}>0$, there exist real matrices $P_{0}$ and $P_{1}$ such that (29) holds. To show the positive definiteness of $P_{0}$ and $P_{1}$, let $\zeta_{0}, \zeta_{1} \in \mathbb{R}^{n}$ be any nonzero vectors, and $x(0)=\zeta_{0}$, for $\gamma(0)=0$, and $x(0)=\zeta_{1}$, for $\gamma(0)=1$, namely, $X_{0}(0)=\zeta_{0} \zeta_{0}^{T}$ and $X_{1}(0)=\zeta_{1} \zeta_{1}^{T}$. Then, by some manipulations, Lemma 5, and (29), we obtain that

$$
\left[\begin{array}{l}
\zeta_{0} \\
\zeta_{1}
\end{array}\right]^{T} \operatorname{diag}\left(P_{0}, P_{1}\right)\left[\begin{array}{l}
\zeta_{0} \\
\zeta_{1}
\end{array}\right]
$$

$$
\begin{aligned}
& =\hat{\varphi}^{T}\left(P_{0}, P_{1}\right) \hat{\varphi}\left(X_{0}(0), X_{1}(0)\right) \\
& =\hat{\varphi}^{T}\left(Q_{0}, Q_{1}\right)\left[I-\lambda \mathcal{M}^{T}(\alpha, \beta)\right]^{-T} \hat{\varphi}\left(X_{0}(0), X_{1}(0)\right) \\
& =\hat{\varphi}^{T}\left(Q_{0}, Q_{1}\right) \sum_{k=0}^{\infty}(\lambda \mathcal{M}(\alpha, \beta))^{k} \hat{\varphi}\left(X_{0}(0), X_{1}(0)\right) \\
& =\sum_{k=0}^{\infty} \lambda^{k} \hat{\varphi}^{T}\left(Q_{0}, Q_{1}\right) \hat{\varphi}\left(X_{0}(k), X_{1}(k)\right) \\
& =\sum_{k=0}^{\infty} \lambda^{k}\left[\varphi^{T}\left(Q_{0}\right) \varphi\left(X_{0}(k)\right)+\varphi^{T}\left(Q_{1}\right) \varphi\left(X_{1}(k)\right)\right] .
\end{aligned}
$$

It can be shown easily that

$$
\begin{aligned}
\varphi^{T} & \left(Q_{0}\right) \varphi\left(X_{0}(k)\right) \\
& =\varphi^{T}\left(Q_{0}\right) \mathbf{E}\{x(k) \otimes x(k)\} \times \mathbf{1}_{\{\gamma(k)=0\}} \\
& =\mathbf{E}\left\{x^{T}(k) Q_{0} x(k)\right\} \times \mathbf{1}_{\{\gamma(k)=0\}} \geq 0 .
\end{aligned}
$$

Similarly, for $\gamma(k)=1$

$$
\varphi^{T}\left(Q_{1}\right) \varphi\left(X_{1}(k)\right) \geq 0 .
$$

In addition, when $k=0$

$$
\varphi^{T}\left(Q_{0}\right) \varphi\left(X_{0}(0)\right)+\varphi^{T}\left(Q_{1}\right) \varphi\left(X_{1}(0)\right)>0 .
$$

With (30)-(33), we obtain that for any nonzero $\zeta_{0}, \zeta_{1} \in \mathbb{R}^{n}$

$$
\left[\begin{array}{l}
\zeta_{0} \\
\zeta_{1}
\end{array}\right]^{T} \operatorname{diag}\left(P_{0}, P_{1}\right)\left[\begin{array}{l}
\zeta_{0} \\
\zeta_{1}
\end{array}\right]>0
$$

which implies $P_{0}>0$ and $P_{1}>0$. This completes the proof.

Corollary 1: Assume that the missing data process $\gamma(k)$ is Bernoulli with the probability distribution (2), then, for a prescribed scalar $\lambda \geq 1$, the closed-loop system in (8) with $\Delta K \equiv 0$ is $\lambda$-exponentially stable if and only if there exist real matrices $P_{0}>0$ and $P_{1}>0$ such that

$$
\begin{aligned}
\lambda A^{T} \hat{P} A-P_{0} & <0 \\
\lambda(A+B K)^{T} \hat{P}(A+B K)-P_{1} & <0
\end{aligned}
$$

where $\hat{P}=(1-\alpha) P_{0}+\alpha P_{1}$.

Remark 2: The presented results can also be extended to linear systems with uncertainties either in the system matrices or in the recovery rate $\alpha$ and the failure rate $\beta$. The reason why we only consider the nominal case is just to make the theory more understandable and to avoid unnecessarily complicated notations.

Remark 3: When choosing different $\alpha$ and $\beta$, (9) and (10) will reduce to some conventional stability criteria. For $\alpha=1, \beta=0$, which corresponds the case without missing data, it can be shown easily that (9) and (10) are equivalent to

$$
\lambda(A+B K)^{T} P(A+B K)-P<0, \quad P>0
$$

which is just the conventional $\lambda$-exponential stability criterion of the closed-loop system. Likewise, when $\alpha=0, \beta=1$, which corresponds the case without the control input, (9) and (10) are equivalent to

$$
\lambda A^{T} P A-P<0, \quad P>0
$$

which coincides with the conventional $\lambda$-exponential stability criterion of the open-loop system.

Remark 4: It should be emphasized that, generally speaking, the controller designed for certain $\alpha_{0}$ and $\beta_{0}$ cannot guarantee the $\lambda$-exponential stability of the closed-loop system for any $1 \geq \alpha \geq \alpha_{0}$ and 
$0 \leq \beta \leq \beta_{0}$, which means that the chance of missing data is reduced. To see this, let us consider a discrete-time linear system with

$$
\begin{aligned}
A & =\left[\begin{array}{cc}
1.1 & 3.0 \\
0 & -1.0
\end{array}\right], \quad B=\left[\begin{array}{ll}
1 & 4 \\
2 & 3
\end{array}\right] \\
K & =\left[\begin{array}{cc}
0.8794 & 2.6527 \\
-0.4508 & -1.4119
\end{array}\right] .
\end{aligned}
$$

It can be shown easily that the closed-loop system with $\alpha_{0}=0.55$ and $\beta_{0}=0.58$ is 1.05 -exponentially stable. However, for $\alpha=1$ and $\beta_{0}=0.58$, the closed-loop system becomes unstable. Fig. 1 gives a complete stability characterization on $\alpha$ and $\beta$.

Remark 5: From the analysis in Remark 3, one may concern under what conditions the controller can guarantee the $\lambda$-exponential stability of the closed-loop system for any $\alpha_{m} \leq \alpha \leq \alpha_{M}$ and $\beta_{m} \leq \beta \leq \beta_{M}$. Actually, it is not difficult to prove that the stable region is a convex set. Therefore, a necessary and sufficient condition for the $\lambda$-exponential stability of the closed-loop system for any $\alpha_{m} \leq \alpha \leq \alpha_{M}$ and $\beta_{m} \leq \beta \leq \beta_{M}$ is that the closed-loop system is $\lambda$-exponentially stable at the vertex $\left(\alpha_{m}, \beta_{M}\right),\left(\alpha_{m}, \beta_{m}\right),\left(\alpha_{M}, \beta_{M}\right)$ and $\left(\alpha_{M}, \beta_{m}\right)$. Moreover, one may ask how to design a controller matrix $K$ independent of the missing data process $\gamma(k)$ such that the closed-loop system is $\lambda$-exponentially stable for any $\alpha_{m} \leq \alpha \leq \alpha_{M}$ and $\beta_{m} \leq \beta \leq \beta_{M}$ with specified $\alpha_{m}, \alpha_{M}, \beta_{m}$ and $\beta_{M}$, and how to further minimize $\alpha_{m}$, $\beta_{m}$ and to maximize $\beta_{M}, \alpha_{M}$. These may consist of some interesting problems for further study.

\section{CONTROLler Synthesis}

In this section, we turn to investigate the design problem of exponential stabilization with uncertain $K$. We provide a sufficient condition for the existence of a desired $K$ in the following theorem.

Theorem 2: For a prescribed scalar $\lambda \geq 1$, if there exist real matrices $X_{0}>0, X_{1}>0, L, Q_{j}>0(j=1,2, \ldots, n)$ and scalars $\epsilon>0$, $b>0$ such that

$$
\begin{aligned}
& X_{1}+X_{1} \geq(1+b) I \\
& {\left[\begin{array}{cc}
-b I & H_{j} X_{1} \\
X_{1} H_{j} & -Q_{j}
\end{array}\right] \leq 0} \\
& {\left[\begin{array}{ccc}
-X_{0} & \# & \# \\
A X_{0} & -\frac{1}{\lambda(1-\alpha)} X_{0} & \# \\
A X_{0} & 0 & -\frac{1}{\lambda \alpha} X_{1}
\end{array}\right]<0}
\end{aligned}
$$

and (37), shown at the bottom of the page, where $\Psi=\operatorname{diag}\left(\bar{\delta}_{11}, \bar{\delta}_{12}, \ldots, \bar{\delta}_{1 m}\right)$ and $\Lambda=\left[E_{1} L E_{2} L \cdots E_{m} L\right]^{T}$, then the closed-loop system (8) with

$$
K=L X_{1}^{-1}
$$

is reliably $\lambda$-exponentially stable.

Proof: Since $0 \leq\left(I-X_{1}\right)\left(I-X_{1}\right)=I-X_{1}-X_{1}+$ $X_{1} X_{1}$, we obtain from (34) that $X_{1} X_{1} \geq b I$. It follows from (35) that $H_{j} X_{1} Q_{j}^{-1} X_{1} H_{j} \leq b I$. Hence

$$
H_{j} X_{1} Q_{j}^{-1} X_{1} H_{j} \leq X_{1} X_{1} \text {. }
$$

According to Theorem 1, the closed-loop system (8) is $\lambda$-exponentially stable if and only if there exist $P_{0}>0$ and $P_{1}>0$ such that

$$
\begin{aligned}
\lambda A^{T} \hat{P}_{0} A-P_{0} & <0 \\
\lambda[A+B(K+\Delta K)]^{T} \hat{P}_{1}[A+B(K+\Delta K)]-P_{1} & <0
\end{aligned}
$$

which, by Schur complement equivalence [25], are equivalent to (36) and

$$
\left[\begin{array}{ccc}
-X_{1} & \# & \# \\
{[A+B(K+\Delta K)] X_{1}} & -\frac{1}{\lambda \beta} X_{0} & \# \\
{[A+B(K+\Delta K)] X_{1}} & 0 & -\frac{1}{\lambda(1-\beta)} X_{1}
\end{array}\right]<0
$$

where $X_{0}=P_{0}^{-1}, X_{1}=P_{1}^{-1}$. Hence, we only need to prove (40). With (38) and the uncertainty form in (6), (40) can be re-written as

$$
\mathcal{G}+\mathcal{B} \Phi \mathcal{L}+(\mathcal{B} \Phi \mathcal{L})^{T}+\sum_{i=1}^{m} \sum_{j=1}^{n} \delta_{i j}\left[\mathcal{K}_{i j} \mathcal{I}+\left(\mathcal{K}_{i j} \mathcal{I}\right)^{T}\right]<0
$$

where $\Phi$ is defined in (7), and

$$
\begin{aligned}
\mathcal{G} & =\left[\begin{array}{ccc}
-X_{1} & \# & \# \\
A X_{1}+B L & -\frac{1}{\lambda \beta} X_{0} & \# \\
A X_{1}+B L & 0 & -\frac{1}{\lambda(1-\beta)} X_{1}
\end{array}\right] \\
\mathcal{K}_{i j} & =\left[\begin{array}{lll}
0 & \left(B E_{i} K H_{j} X_{1}\right)^{T} & \left(B E_{i} K H_{j} X_{1}\right)^{T}
\end{array}\right]^{T} \\
\mathcal{B} & =\left[\begin{array}{lll}
0 & B^{T} & B^{T}
\end{array}\right]^{T}, \quad \mathcal{L}=\left[\begin{array}{lll}
L & 0 & 0
\end{array}\right] \\
\mathcal{I} & =\left[\begin{array}{lll}
I & 0 & 0
\end{array}\right] .
\end{aligned}
$$

By (5) and using Lemma 4, we obtain that (41) holds if the following inequality holds for some $\epsilon>0, Q_{j}>0(j=1,2, \ldots, n)$ :

$$
\begin{aligned}
\mathcal{G}+\epsilon \mathcal{B} \Psi^{2} \mathcal{B}^{T}+\epsilon^{-1} \mathcal{L}^{T} \mathcal{L}+m & \sum_{j=1}^{n} \bar{\delta}_{2 j} \mathcal{I}^{T} Q_{j} \mathcal{I} \\
& +\sum_{i=1}^{m} \sum_{j=1}^{n} \bar{\delta}_{2 j} \mathcal{K}_{i j} Q_{j}^{-1} \mathcal{K}_{i j}^{T}<0 .
\end{aligned}
$$

Simple manipulations together with (38) and (39) yield that

$$
\begin{aligned}
\mathcal{K}_{i j} Q_{j}^{-1} \mathcal{K}_{i j}^{T} & \leq\left[\begin{array}{c}
0 \\
B E_{i} K \\
B E_{i} K
\end{array}\right] X_{1} X_{1}\left[\begin{array}{c}
0 \\
B E_{i} K \\
B E_{i} K
\end{array}\right]^{T} \\
& =\left[\begin{array}{c}
0 \\
B E_{i} L \\
B E_{i} L
\end{array}\right]\left[\begin{array}{c}
0 \\
B E_{i} L \\
B E_{i} L
\end{array}\right]^{T} .
\end{aligned}
$$

It follows from (40)-(43), by Schur complement, that (40) holds if (37) holds. This completes the proof.

Remark 6: When $\bar{\delta}_{1 i}$ and $\bar{\delta}_{2 j}$ are known, (34)-(37) are LMIs with respect to variables $X_{0}, X_{1}, L, Q_{j}(j=1,2, \ldots, n), \epsilon$, and $b$, which can be checked by efficient algorithms such as the interior-point method. Then, a desired controller gain can be computed easily from the solutions of the LMIs. The obtained controller does not only stabilize the original system, but also makes the closed-loop system render a desirable decay rate.

$$
\left[\begin{array}{ccccc}
-X_{1}+m \sum_{j=1}^{n} \bar{\delta}_{2 j} Q_{j} & \# & \# & \# & \# \\
A X_{1}+B L & -\frac{1}{\lambda \beta} X_{0}+\epsilon B \Psi^{2} B^{T} & \# & \# & \# \\
A X_{1}+B L & \epsilon B \Psi^{2} B^{T} & -\frac{1}{\lambda(1-\beta)} X_{1}+\epsilon B \Psi^{2} B^{T} & \# & \# \\
L & 0 & 0 & -\epsilon I & \# \\
0 & \left(\sum_{j=1}^{n} \bar{\delta}_{2 j}\right) \Lambda B^{T} & \left(\sum_{j=1}^{n} \bar{\delta}_{2 j}\right) \Lambda B^{T} & 0 & -\left(\sum_{j=1}^{n} \bar{\delta}_{2 j}\right) I
\end{array}\right]<0
$$



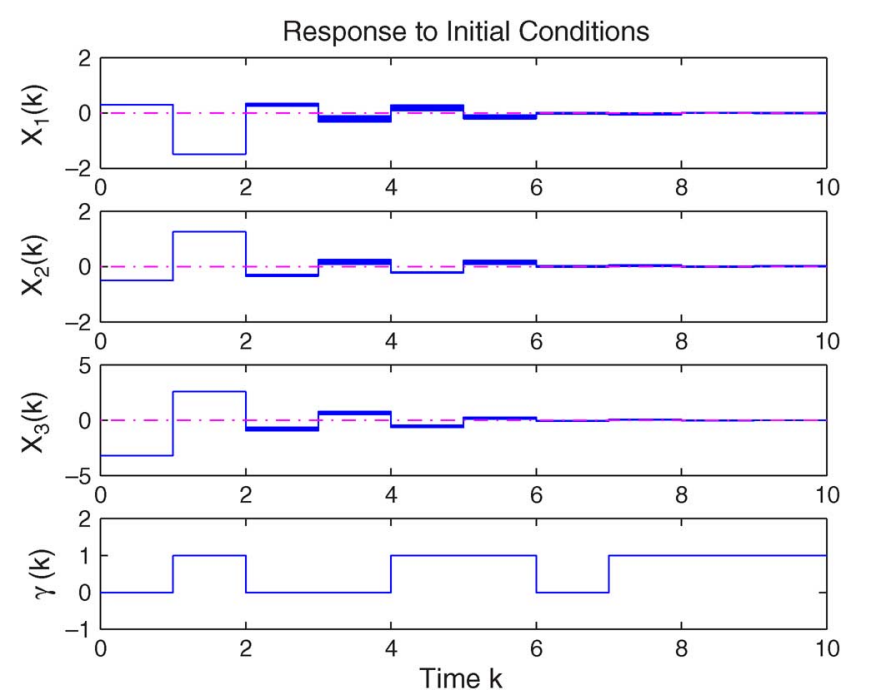

Fig. 2. Closed-loop response to initial condition.

\section{AN ILLUSTRATIVE EXAMPLE}

Consider a discrete-time linear system whose control signals are transmitted through a fading channel with packet loss:

$$
x(k+1)=\left[\begin{array}{ccc}
1.2 & 0.5 & 0.5 \\
0 & 0.7 & -0.5 \\
0.1 & 0 & -0.8
\end{array}\right] x(k)+\gamma(k)\left[\begin{array}{cc}
0 & 1.5 \\
0 & 1 \\
1 & 0
\end{array}\right] u(k) .
$$

It is assumed that, for the packet loss process $\gamma(k), \alpha=0.7$ and $\beta=$ 0.15 . Similar models have appeared in [12], [15], [26]. The uncertainty bounds in the controller are given as

$$
\begin{aligned}
& \bar{\delta}_{21}=0.02, \quad \bar{\delta}_{22}=0.05, \quad \bar{\delta}_{23}=0.06 \\
& \bar{\delta}_{11}=\bar{\delta}_{12}=0.1 .
\end{aligned}
$$

For a prescribed $\lambda=1.6$, it is easy to check, by using the MATLAB LMI Toolbox, that (34)-(37) are feasible, and a non-fragile controller gain is obtained as

$$
K=\left[\begin{array}{ccc}
-0.2417 & -0.0303 & 0.4159 \\
-0.4643 & -0.4240 & -0.0223
\end{array}\right] \text {. }
$$

The resulting closed-loop system is not only stable, but also has a decay rate larger than 1.6. To show the reliability of the designed controller, we take 20 samples randomly on the uncertain controller $K+\Delta K$ with uncertainties described in (6), and compute the response for each sample. Fig. 2 gives the response of the 20 sampled closed-loop systems to the initial condition $x(0)=\left[\begin{array}{lll}0.3 & -0.5 & -3.2\end{array}\right]^{T}$.

\section{CONCLUSION}

In this technical note, we have studied the exponential stabilization problem for a class of discrete-time linear systems with uncertainties in controllers and missing data in actuators. A necessary and sufficient condition, which does not only guarantee the stability of the closed-loop system but also gives a lower bound on the decay rate, is established in terms of LMIs. Based on this condition, an LMI-based approach is provided to design a state-feedback controller such that the closed-loop system is exponentially stable with a prescribed lower bound on the decay rate for all admissible uncertainties in the controller and the known missing data process in the actuator. The obtained re- sults can also be extended to the case with uncertainties either in the system matrices or in the recovery rate and the failure rate.

\section{REFERENCES}

[1] G. Garcia, J. Bernussou, and D. Arzelier, "Robust stabilization of discrete-time linear systems with norm-bounded time-varying uncertainty," Syst. Control Lett., vol. 22, no. 5, pp. 327-339, May 1994.

[2] P. Myszkorowski, "Robust control of linear discrete-time systems," Syst. Control Lett., vol. 22, no. 4, pp. 277-280, Apr. 1994.

[3] S. Xu, J. Lam, and C. Yang, "Quadratic stability and stabilization of uncertain linear discrete-time systems with state delay," Syst. Control Lett., vol. 43, no. 2, pp. 77-84, Jun. 2001.

[4] C. Ferreiraa and F. C. Silva, "On the stabilization of linear discrete-time systems," Linear Algebra Appl., vol. 390, pp. 7-18, Oct. 2004.

[5] J. Yee, G. Yang, and J. Wang, "Non-fragile guaranteed cost control for discrete-time uncertain linear systems," Int. J. Syst. Sci., vol. 32, no. 7, pp. 845-853, Jul. 2001.

[6] G.-H. Yang, J. L. Wang, and Y. C. Soh, "Guaranteed cost control for discrete-time linear systems under controller gain perturbations," Linear Algebra Appl/, vol. 312, no. 1-3, pp. 161-180, Jun. 2000.

[7] D. Yue and J. Lam, "Non-fragile guaranteed cost control for uncertain descriptor systems with time-varying state and input delays," Optim. Control Appl. Meth., vol. 26, no. 2, pp. 85-105, Mar./Apr. 2005.

[8] S. Xu, J. Lam, G. H. Yang, and J. L. Wang, "Stabilization and $\mathcal{H}_{\infty}$ control for uncertain stochastic time-delay systems via non-fragile controllers," Asian J. Control, vol. 8, no. 2, pp. 197-200, Jun. 2006.

[9] E. N. Gilbert, "Capacity of a burst-noise channel," Bell Syst. Tech. J., vol. 39, pp. 1253-1265, Sep. 1960.

[10] E. O. Elliott, "Estimates of error rates for codes on burst-noise channels," Bell Syst. Tech. J., vol. 42, pp. 1977-1997, Sep. 1963.

[11] Z. Wang, D. W. C. Ho, and X. Liu, "Variance-constrained filtering for uncertain stochastic systems with missing measurements," IEEE Trans. Automat. Control, vol. 48, no. 7, pp. 1254-1258, Jul. 2003.

[12] S. C. Smith and P. Seiler, "Estimation with lossy measurements: Jump estimators for jump systems," IEEE Trans. Automat. Control, vol. 48, no. 12, pp. 2163-2171, Dec. 2003.

[13] Z. Wang, F. Yang, D. W. C. Ho, and X. Liu, "Robust $\boldsymbol{H}_{\infty}$ filtering for stochastic time-delay systems with missing measurements," IEEE Trans. Signal Processing, vol. 54, no. 7, pp. 2579-2586, Jul. 2006.

[14] H. Gao and T. Chen, " $\mathcal{H}_{\infty}$ estimation for uncertain systems with limited communication capacity," IEEE Trans. Automat. Control, vol. 52, no. 11, pp. 2070-2084, Nov. 2007.

[15] S. Hu and W.-Y. Yan, "Stability robustness of networked control systems with respect to packet loss," Automatica, vol. 43, no. 7, pp. 1243-1248, Jul. 2007.

[16] G. H. Yang and J. L. Wang, "Non-fragile $\mathcal{H}_{\infty}$ control for linear systems with multiplicative controller gain variations," Automatica, vol. 37, no. 5, pp. 727-737, May 2001.

[17] J. Ackermann, Sampled-Data Control Systems. Berlin, Germany: Springer, 1985.

[18] D. Famularo, C. T. Abdallah, A. Jadbabaie, P. Dorato, and W. M. Haddad, "Robust non-fragile LQ controllers: The static state feedback case," in Proc. Amer. Control Conf., Jun. 1998, vol. 2, pp. 1109-1113.

[19] K. J. Åström and B. Wittenmark, Computer Controlled Systems: Theory and Design. Englewood Cliffs, NJ: Prentice-Hall, 1997.

[20] A. W. Naylor and G. R. Sell, Linear Operator Theory in Engineering and Science. New York: Springer-Verlag, 1982.

[21] O. L. V. Costa and M. D. Fragoso, "Stability results for discrete-time linear systems with $\{\mathrm{M}\}$ arkovian jumping parameters," J. Math. Anal. Appl., vol. 179, no. 1, pp. 154-178, Oct. 1993.

[22] I. R. Petersen, "A stabilization algorithm for a class of uncertain linear systems," Syst. Control Lett., vol. 8, no. 4, pp. 351-357, Mar. 1987.

[23] Y. Wang, L. Xie, and C. E. de Souza, "Robust control of a class of uncertain nonlinear systems," Syst. Control Lett., vol. 19, no. 2, pp. 139-149, Aug. 1992.

[24] A. J. Laub, Matrix Analysis for Scientists \& Engineers. Philadelphia, PA: SIAM, 2005.

[25] S. Boyd, L. El Ghaoui, E. Feron, and V. Balakrishnan, Linear Matrix Inequalities in System and Control Theory. Philadelphia, PA: Society for Industrial and Applied Mathematics (SIAM), 1994.

[26] M. Huang and S. Dey, "Stability of Kalman filtering with Markovian packet losses," Automatica, vol. 43, no. 4, pp. 598-607, Apr. 2007. 